\title{
Convective motion in collisionless trapped electron mode turbulence
}

\author{
Y. Xiao and Z. Lin \\ Department of Physics and Astronomy, University of California, Irvine, CA 92697, U.S.A. \\ Date: $1 / 06 / 2011$
}

\begin{abstract}
Global gyrokinetic particle simulation of collisionless trapped electron mode turbulence in toroidal plasmas finds both diffusive and convective electron motion using a Lagrangian analysis. The convective motion is identified using simulation and analytic theory to arise from the conservation of the second invariant when resonant trapped electrons lose kinetic energy to the drift wave. A resonance broadening model fits well the diffusive and convective electron motion.
\end{abstract}

Collisionless trapped electron mode (CTEM) turbulence [1] is a prominent candidate for the anomalous transport observed in high temperature fusion plasma. Understanding the transport mechanism of CTEM is critical to improve the electron confinement [2-4] of a burning plasma such as ITER [5] where the energetic $\alpha$-particles will mostly heat electrons. Recently the large scale gyrokinetic particle simulation has emerged as a major tool to study the CTEM turbulent transport [6, 7]. A latest study finds that the electron transport contains a significant non-diffusive/ballistic component, which is due to the weak detuning of the toroidal precessional resonance and the existence of mesoscale turbulence eddies [8]. This nondiffusive component was further confirmed by the nonGaussian statistics of the electron heat fluxes [9]. However, the kinetic origin of this non-diffusive component remains elusive. In this letter, we identify the existence of a convective motion of the trapped electrons in the CTEM turbulence by a Lagrangian analysis. Our gyrokinetic simulation and analytic theory find that this convection effect arises because of the conservation of the second invariant for the trapped electrons. The electron phase diagram is made for both convection and diffusion motion in the space of pitch angle and energy, which shows that the convection motion could be important for both deeply trapped and barely trapped electrons, while the diffusion is dominated by the deeply trapped electrons. A resonance broadening model is found to fit well for the diffusion motion, and reasonably well for the convection motion.

Simulation Description.---We carried out a gyrokinetic particle simulation using the GTC code [10] to study the CTEM turbulence based on the following parameters [9]: $R / L_{T e}=6.9, R / L_{T i}=2.2, R / L_{n}=2.2, a=250 \rho_{i}, a / R_{0}=0.358, T_{i}=T_{e} \equiv T, m_{i} / m_{e}=1837$, the safety factor $q=0.58+1.09 r / a+1.09(r / a)^{2}$, with magnetic shear $s \equiv d \ln q / d \ln r=0.78$ at $r=0.5 a$. The ion gyroradius $\rho_{i}=v_{i} / \Omega_{i}$ with $v_{i}=\sqrt{T_{i} / m_{i}}$. A circular cross section model is used for the equilibrium magnetic field, $B=B_{0} /\left(1+r \cos \theta / R_{0}\right)$ with the simulation carried out in the annulus between $r=0.1 a$ and $r=0.9 a$. The ion is treated by the gyrokinetic equation [11] and electron by an 
electrostatic version of the fluid-kinetic hybrid model [12]. The electron heat flux $q_{e}$ is represented by an effective heat conductivity $\chi_{e}$ using to the simple relation $q_{e}=n_{0} \chi_{e} \nabla T_{e}$, although it will be clarified later that a more appropriate expression for the heat flux includes both a diffusive and a convective term. The heat conductivity $\chi_{e}$ and the volume averaged turbulence amplitude $\left(|\delta \phi|=\sqrt{\left\langle\delta \phi^{2}\right\rangle}\right)$ are shown in Fig. 1(a). After an initial exponential growth driven by the CTEM instability, the turbulent transport saturates at a level of gyroBohm diffusivity $\chi_{G B}=\rho_{i}^{2} v_{i} / a$. However, there is a transient period at $t \subset[20,60] R_{0} / v_{i}$, where the turbulence amplitude is not fully saturated. The turbulence amplitude is fully saturated in the following period of $[60,100] R_{0} / v_{i}$. which is sufficiently long to cover all physical time scales relevant to the turbulent transport dynamics [8].

Lagrangian Analysis.---In the simulation, over 1.4 million electrons are tracked in the simulation as test particles, which are initially selected from a very thin annulus centred at $r=0.5 a$ with width less than one typical eddy size [9], in a way such that they are evenly distributed in the 2D phase space $(\lambda, E)$, with the kinetic energy $E=m_{e} v^{2} / 2$ at $t=0$ and the initial pitch angle $\lambda=\mu \mathrm{B}_{0} / E$. There are about 3200 particles tracked at each phase grid point, which can effectively reduce the Monte Carlo sampling noise. We note that these electrons are actually the self-consistent markers in the particle simulation. At each grid point in the $(\lambda, E)$ phase space, we compute the mean radial drift $\Delta r$ and diffusion $\Delta r^{2}$ for these test particles at each time step using the following formula:

$$
\begin{gathered}
\Delta r(t)=\frac{1}{N} \sum_{i=1}^{N} \delta r_{i}(t) \equiv\left\langle\delta r_{i}(t)\right\rangle, \\
\Delta r^{2}(t)=\frac{1}{N} \sum_{i=1}^{N}\left(\delta r_{i}(t)-\left\langle\delta r_{i}(t)\right\rangle\right)^{2},
\end{gathered}
$$

where $\delta r_{i}(t)=r_{i}(t)-r_{i}(0)$, and $i$ is the particle label. As shown by Fig. 1(b), both $\Delta r$ and $\Delta r^{2}$ increase with time and the trapped electrons possess a radially outward drift. A linear fit of these two quantities in the fully saturated turbulence gives the convection velocity $v_{p}$ and the diffusion coefficient $D$ : $v_{p}=\Delta r / \Delta t$ and $D=\Delta r^{2} / 2 \Delta t$. These two quantities are then computed for each phase space grid, as shown in Fig. 2(a) and 3(a). These results show that both diffusion and convection make significant contribution to the electron transport in the CTEM turbulence. This is in contrast to the ion transport in the ion temperature gradient (ITG) driven turbulence, which is dominated by the diffusive component [13] due to parallel wave-particle decorrelation [9, 14].

Diffusion.--- In Fig. 2(a), for each energy $E$, the diffusion is stronger for deeply trapped electrons (larger $\lambda$ ) than the barely trapped electrons (smaller $\lambda$ ). For each pitch angle $\lambda$, there is an energy that maximizes the diffusion, which is found to be the kinetic energy that gives rise to the precessional resonance. The toroidal precessional frequency of a trapped electron in the large aspect ratio limit is given by [15]

$$
\omega_{\text {pres }}=\frac{v_{i}}{R_{0}} \frac{q \rho_{i}}{r}(2-\lambda) \frac{2 E}{T_{i}}\left[\frac{\mathbf{E}(k)}{\mathbf{K}(k)}-\frac{1}{2}+2 s\left(\frac{\mathbf{E}(k)}{\mathbf{K}(k)}+k-1\right)\right],
$$

with $\mathbf{K}(k)$ and $\mathbf{E}(k)$ the complete elliptic functions, and $k=(1+\varepsilon-\lambda) / 2 \varepsilon$, where $\varepsilon=r / R_{0}$ is the inverse aspect ratio, and $\lambda \subset[1-\varepsilon, 1+\varepsilon]$ for trapped electrons. Then each pitch angle $\lambda$ and energy $E$ corresponds to a toroidal precessional frequency $\omega_{\text {pres }}$. Thus we can transfer the 2D diffusion 
distribution function $D(\lambda, E)$ in Fig. 2(a) to a $1 \mathrm{D}$ function $D\left(\omega_{\text {pres }}\right)$ in Fig. 2(b). The initial values $q=1.4, s=0.78$ and $r=0.5 a$ are used to calculate $\omega_{\text {pres }}$ by assuming $q$ and $s$ do not change much when the particles move radially during this simulation period. As shown in Fig. 2(a), the diffusion is most significant for $\omega_{\text {pres }} \subset[0.020,0.048]$.

The resonant trapped electrons are expected to make dominant contribution to the diffusion. Here we consider the resonance between the drift wave and the electron banana center executing toroidal precession. Therefore, $\left(\zeta, \omega_{\text {pres }}\right)$ is the pair of the canonical variables for this precessional resonance. Consequently, a Lorentzian distribution based on the resonance broadening formula [16]

$$
D\left(\omega_{\text {pres }}\right)=\frac{D_{0} \Delta \omega_{\text {pres }}^{2}}{\left(\omega_{\text {pres }}-\omega_{0}\right)^{2}+\Delta \omega_{\text {pres }}^{2}},
$$

is found to well fit the $1 \mathrm{D}$ diffusion distribution $D\left(\omega_{\text {pres }}\right)$, which gives $\omega_{0}=0.0345 v_{i} / R_{0}$ and $\Delta \omega_{\text {pres }}=0.0282 v_{i} / R_{0}$. On the other hand, the linear eigen frequency of the CTEM instability is dispersiveless [17], $\omega_{r}^{\text {lin }}=1.52 k_{\theta} \rho_{i} v_{i} / R_{0}$, which gives $\omega_{r}^{\text {lin }} / n=0.034 v_{i} / R_{0}$ (almost identical to the fitting value $\omega_{0}$ !). The nonlinear CTEM turbulence intensity peaks at $k_{\theta} \rho_{i} \approx 0.35$ [9], at which the toroidal mode number $n \approx 31$ and the linear growth rate $\gamma^{\text {Lin }}=0.21 v_{i} / L_{n}$ [17]. Therefore, $\gamma^{\text {lin }} / n=0.030 v_{i} / R_{0}$, which is roughly $10 \%$ difference from the fitting value $\Delta \omega_{\text {pres }}$. The dashed line in Fig. 2(b) is the Eq. (4) by using the linear eigen frequency $\omega_{r}^{\text {lin }} / n$ and growth rate $\gamma^{\text {lin }} / n$. Therefore, the Lorentzian model is very accurate to describe the diffusion part of the electron transport in the CTEM turbulence.

Outward Convection.---The 2D contour of Fig. 3(a) shows that there is an outward convection for the trapped electrons in the CTEM turbulence. The barely trapped electrons contribute more to the convection than the deeply trapped electrons. However, the convection velocity does not decrease monotonically with the pitch angle $\lambda$. In fact it first deceases with $\lambda$ for the barely trapped electrons and increases with $\lambda$ for the deeply trapped electrons. The 2D convection velocity distribution $V_{p}(\lambda, E)$ in Fig. 3(a) can be similarly transformed to a $1 \mathrm{D}$ distribution function $V_{p}\left(\omega_{\text {pres }}\right)$ according to Eq. (3). As shown in Fig. 3(b), the distribution function $V_{p}\left(\omega_{\text {pres }}\right)$ (the red dots) can be roughly fitted by a Lorentzian of Eq. (4) for the barely trapped electron, with the peak frequency $\omega_{0}=0.033 v_{i} / R_{0}$ and spectral width $\Delta \omega_{\text {pres }}=0.020 v_{i} / R_{0}$. Hence the Lorentzian in Eq. (4) fits reasonably well for the barely trapped electrons except that the spectral width is slightly smaller than the linear growth rate. On the other hand, the Lorentzian fit of the distribution function $V_{p}\left(\omega_{\text {pres }}\right)$ for the deeply trapped electrons gives the peak frequency $\omega_{0}=0.042 v_{i} / R_{0}$, which is slightly higher than the linear eigen frequency $\omega_{r} / n=0.034 v_{i} / R_{0}$. This can also be confirmed by Fig. 3(a), where the curve $\omega_{\text {pres }}=0.034$ does not exactly go through the peak value of the deeply trapped convection velocities.

The outward convection is found to arise from the conservation of the second invariant $J_{\|} \equiv m_{e} \oint d l v_{\|}=J(\lambda, E, r)$, where $r$ is essentially the banana tip since electron banana width is negligibly small compared to the turbulence eddy size [9]. Since the mode frequency $\omega$ is smaller than the electron bounce frequency $\omega_{b}$, the second invariant $J_{\|}$is conserved in the CTEM turbulence.

Then $\Delta J_{\|}=0$ gives 


$$
\Delta r / R_{0}=-F(\lambda, E, r) \Delta E / T_{i},
$$

with the pinch coefficient $F(\lambda, E, r) \equiv \frac{T_{i}}{R_{0}} \frac{\partial J_{\|} /\left.\partial E\right|_{\mu, r}}{\partial J_{\|} /\left.\partial r\right|_{\mu, E}}$. Therefore, the convection velocity $V_{p}=\frac{d \Delta r}{d t}=-R_{0} F(\lambda, E, r) \frac{d}{d t}\left(\frac{\Delta E}{T_{i}}\right)$. Thus, the convection velocity is related to the energy change of the electrons. In the CTEM turbulence, the trapped electrons lose kinetic energy to the unstable drift waves, which will introduce an outward convection if the coefficient $F(\lambda, E, r)>0$. Note that the toroidal precessional frequency [18] of trapped electrons $\omega_{\text {pres }}=\frac{q \rho_{i} v_{i}}{r T_{i}} \frac{\partial J_{\|} /\left.\partial r\right|_{\mu, E}}{\partial J_{\|} /\left.\partial E\right|_{\mu, r}}$, then the convection coefficient is given by a simple relation

$$
F(\lambda, E, r)=\frac{q \rho_{i}}{r} \frac{v_{i}}{R_{0} \omega_{\text {pres }}},
$$

with $\omega_{\text {pres }}>0$ in this normal magnetic shear case $(s \approx 0.78)$.

The 2D contour of the energy loss rate $(-d \Delta E / d t)$ is shown in Fig. 4(a) for each phase grid, which is calculated from the simulation data. Indeed we see an energy loss for trapped electrons, as it should be. The similarity between Fig. 4(a) and Fig. 3(a) confirms the outward electron convection is due to the loss of electron kinetic energy to the drift waves.

The convection coefficient $F(\lambda, E, r)$ can be computed from Fig. 3(a) and Fig. 4(a), as shown by the discrete markers in Fig. 4 (b). The solid curve in Fig. 4(b) is taken from the analytic formula, Eq. (5). The simulation data agree with the theory in a quantitatively satisfactory way, especially at high energy where the $E^{-1}$ scaling is directly coming from $\omega_{\text {pres }} \propto E$.

Therefore, the convective motion of trapped electrons is purely due to the conservation of the second invariant $J_{\|}$, which has been proved by quantitatively comparing simulation and theory. The energy exchange direction between the particle and drift wave, plus the sign of the convection coefficient $F(\lambda, E, r)$, determines the direction of the convection.

Summary and Discussion.---We found that the trapped electrons have both diffusive and convective motion in the CTEM turbulence which can be described by a resonance broadening model. A predictive electron turbulent transport model should contain both terms. This convection motion of the trapped electrons is caused by the loss of kinetic energy and the conservation of the second invariant. In a reverse shear case, Eq. (5) and (6) remain valid, but $\omega_{\text {pres }}<0$ and the trapped electrons can no longer excite instability to lose kinetic energy. This kinetic mechanism of the trapped electron convection is very robust even when the time scale is larger than the eddy rotation time of the observed mesoscale eddies in the CTEM turbulence [8]. Similar transport mechanism is also likely to occur for the energetic particle transport in the fishbone instability [19, 20]. 
Figure lists

Figure 1.
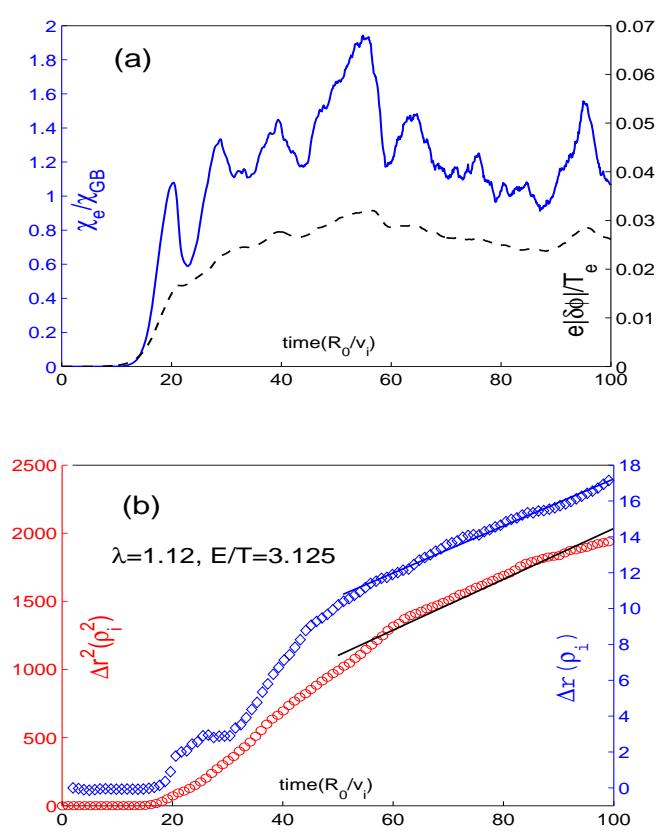

Fig 1. (a): History of electron heat conductivity $\chi_{e}$ and turbulence amplitude, averaged over $r \subset[0.37,0.63] a$. (b): History of mean radial excursion $\Delta r$ and diffusion $\Delta r^{2}$ at one sample phase point. 
Figure 2.
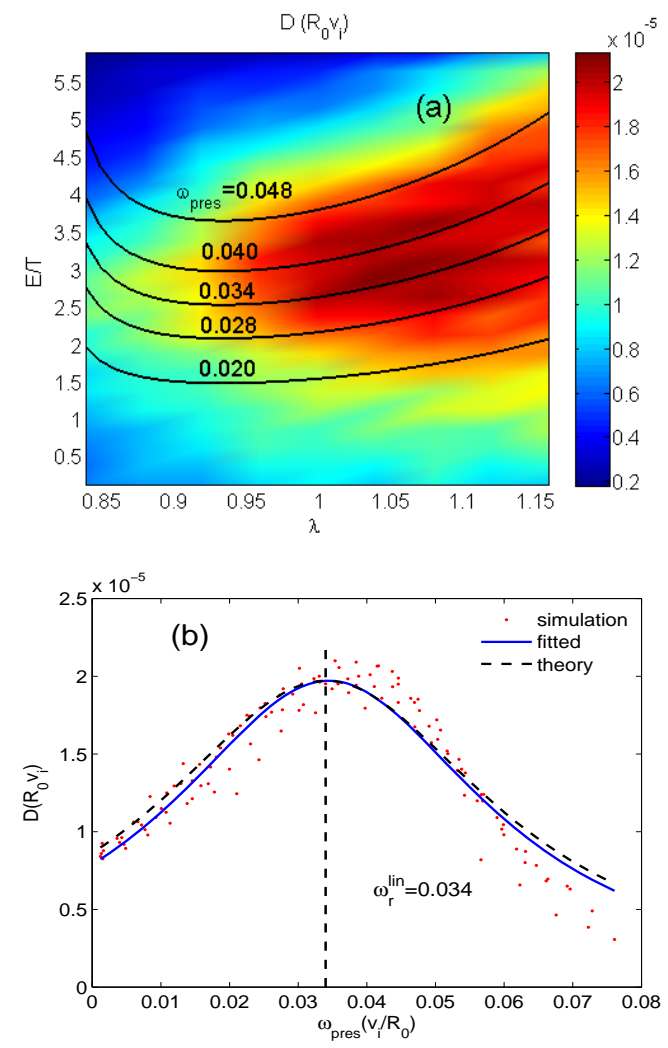

Fig 2. (a): Electron diffusion $D$ in 2D phase space. Bottom panel (b): Electron diffusion $D$ vs electron toroidal precessional frequency (red dots), which is fitted by Eq. (4) (solid line). The dashed line is from Eq. (4) by using the theoretical linear eigen frequencies. 


\section{Figure 3}


Fig 3. (a): Outward convection velocity in 2D phase space. (b) Convection velocity vs electron toroidal precessional frequency (red dots) for barely trapped electrons, which is fitted by Eq. (4) (solid line). 
Figure 4.
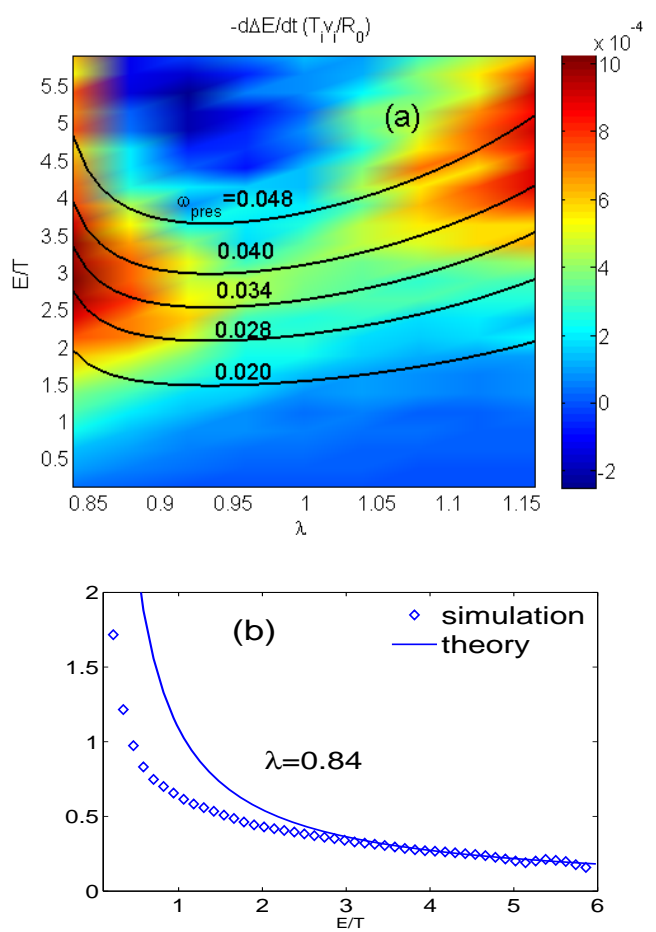

Fig. 4. (a): Electron energy loss rate in 2D phase space. (b) Pinch coefficient $F(\lambda, E, r)$ vs energy $E / T$ at $r=0.5 a$. The discrete diamonds are from the simulation and the solid curves are from analytic formula in Eq. (6).

\section{Acknowledgments}

The work is supported by US DOE SciDac GPS and GSEP centers and an INCITE award. The authors are very grateful to L. Chen, I. Holod and W. Zhang for useful discussions.

\section{References}

1. Horton, W., Drift waves and transport. Reviews of Modern Physics, 1999. 71(3): p. 735-778.

2. DeBoo, J.C., et al., Probing plasma turbulence by modulating the electron temperature gradient. Physics of Plasmas, 2010. 17(5): p. 056105.

3. Garbet, X., et al., Turbulent particle transport in magnetized plasmas. Physical Review Letters, 2003. 91(3).

4. Ryter, F., et al., Experimental study of trapped-electron-mode properties in tokamaks: Threshold and stabilization by collisions. Physical Review Letters, 2005. 95(8).

5. ITER. Available from: http://www.iter.org/.

6. Sydora, R.D., V.K. Decyk, and J.M. Dawson, Fluctuation-induced heat transport results from a large global 3D toroidal particle simulation model. Plasma Physics and Controlled Fusion, 1996. 38(12A): p. A281-A294. 
7. Lang, J.Y., S.E. Parker, and Y. Chen, Nonlinear saturation of collisionless trapped electron mode turbulence: Zonal flows and zonal density. Physics of Plasmas, 2008. 15(5).

8. Xiao, Y. and Z.H. Lin, Turbulent Transport of Trapped-Electron Modes in Collisionless Plasmas. Physical Review Letters, 2009. 103(8).

9. Xiao, Y., et al., Fluctuation characteristics and transport properties of collisionless trapped electron mode turbulence. Physics of Plasmas, 2010. 17(2).

10. Lin, Z., et al., Turbulent transport reduction by zonal flows: Massively parallel simulations. Science, 1998. 281(5384): p. 1835-1837.

11. Lee, W.W., GYROKINETIC APPROACH IN PARTICLE SIMULATION. Physics of Fluids, 1983. 26(2): p. 556-562.

12. Lin, Z., et al., Global gyrokinetic particle simulations with kinetic electrons. Plasma Physics and Controlled Fusion, 2007. 49(12B): p. B163-B172.

13. Zhang, W.L., Z.H. Lin, and L. Chen, Transport of energetic particles by microturbulence in magnetized plasmas. Physical Review Letters, 2008. 101(9).

14. Holod, I. and Z. Lin, Statistical analysis of fluctuations and noise-driven transport in particlein-cell simulations of plasma turbulence. Physics of Plasmas, 2007. 14(3).

15. Helander, P. and D. Sigmar, Collisional Transport in Magnetized Plasmas. 2005: Cambridge University Press.

16. Dupree, T.H., NONLINEAR THEORY OF DRIFT-WAVE TURBULENCE AND ENHANCED DIFFUSION. Physics of Fluids, 1967. 10(5): p. 1049-\&.

17. Rewoldt, G., Z. Lin, and Y. Idomura, Linear comparison of gyrokinetic codes with trapped electrons. Computer Physics Communications, 2007. 177(10): p. 775-780.

18. White, R.B. and M.S. Chance, HAMILTONIAN GUIDING CENTER DRIFT ORBIT CALCULATION FOR PLASMAS OF ARBITRARY CROSS-SECTION. Physics of Fluids, 1984. 27(10): p. 2455-2467.

19. White, R.B., et al., THEORY OF MODE-INDUCED BEAM PARTICLE LOSS IN TOKAMAKS. Physics of Fluids, 1983. 26(10): p. 2958-2965.

20. Chen, L., R.B. White, and M.N. Rosenbluth, EXCITATION OF INTERNAL KINK MODES BY TRAPPED ENERGETIC BEAM IONS. Physical Review Letters, 1984. 52(13): p. 1122-1125. 